\title{
Hätten Sie nicht gern eine Super-MFA?
}

\author{
Dieser MMW-Ausgabe liegt wieder „info praxisteam“ bei, das Magazin für Medizinische Fachangestellte. \\ Ein Hauptthema in Heft 2/17 ist die Möglichkeit der Weiterbildung zur Versorgungsassistentin, die viele \\ Arbeiten selbstständig erledigen kann. Das ist interessant für die Mitarbeiterin - und für die Praxis.
}

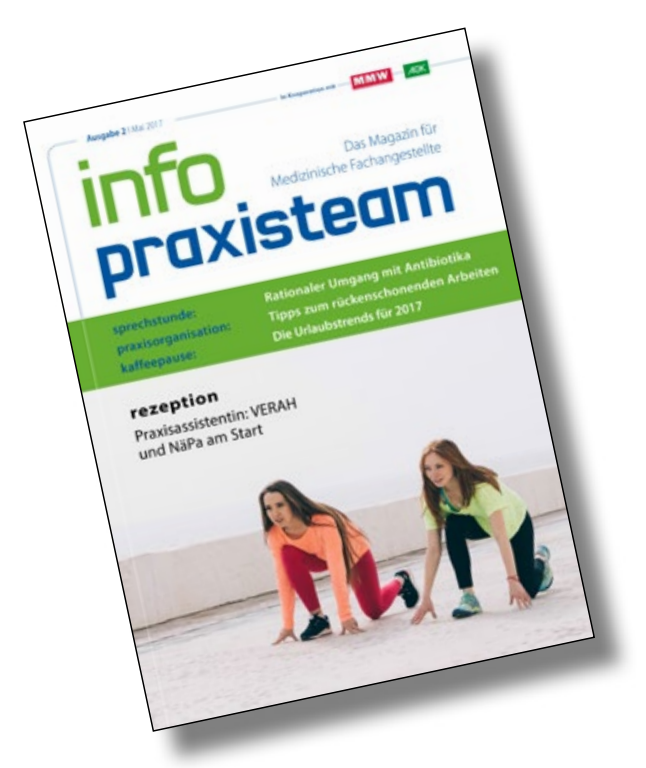

_ Für Medizinische Fachangestellte (MFA) gibt es mittlerweile ein breites Spektrum an strukturierten Fortbildungen. Mehr Verantwortung, eine selbstständige Arbeitsweise und Abwechslung im Praxisalltag verspricht vor allem die Fortbildung zur Praxisassistentin, die delegierbare ärztliche Leistungen übernimmt. „info praxisteam“ informiert über die Weiterbildungsmöglichkeiten zur nichtärztlichen Versorgungsassistentin in der Rubrik „rezeption“.

Während der Deutsche Hausärzteverband gemeinsam mit dem Verband der medizinischen Fachberufe die Fortbildung zur „Versorgungsassistentin in der Hausarztpraxis“ (VERAH) anbieten, hat die Bundesärztekammer die Fortbildung zur „nicht-ärztlichen Praxisassistentin“ (NäPA) entwickelt. Neben diesen beiden bundesweiten Konzepten gibt es regionale Modelle wie „agnes zwei“ in Brandenburg und „EVA“, wie die NäPA in Nordrhein-Westfalen heißt.
Bundesärztekammer und Hausärzteverband haben vereinbart, die im jeweiligen Programm erlangte Qualifikation gegenseitig anzurechnen. Aktutell gibt es deutschlandweit bereits mehr als 9.400 VERAH.

Beide Fortbildungen umfassen die Kompetenzen zur Übernahme delegationsfähiger Leistungen in der ambulanten Versorgung, um den Arzt zu entlasten und auf Anweisung Aufgaben wie selbstständige Hausbesuche und Besuche im Alten- oder Pflegeheim zu übernehmen. Zum Ausbildungsprogramm gehören daher u.a. Blutdruck- und Blutzuckermessungen, das Anlegen des Langzeit-EKG, die Wundversorgung oder die Sturzprophylaxe. Auch standardisierte Testverfahren, etwa den Uhrentest zur Erkennung einer Demenz, kann eine Praxisassistentin selbstständig durchführen.

\section{Mehr Kompetenz, neue Perspektiven}

Das zur Ausbildung gehörige Praktikum kann z. B. in Pflegeheimen, ambulanten Pflegediensten, Hospizen, Pflegestützpunkten oder Apotheken, aber auch in Sanitätshäusern, Haus- und Facharztpraxen oder Medizinischen Versorgungszentren, bei Physiotherapeuten, Logopäden oder Ergotherapeuten abgeleistet werden.

Das eröffnet neue Berufsperspektiven: Direkt nutzbar sind die erworbenen Fachkenntnisse und Kontakte z. B. beim Case Management in der Behandlung chronisch Kranker. Die zur Praxisassistentin geschulten MFA koordinieren dabei alle Dienste, halten engen Kontakt zu den Patienten und pflegen das komplexe Betreuungsnetzwerk.
Auch bei der Antibiotikatherapie sind MFA gefordert - schließlich sind sie häufig erste Anlaufstelle für die Patienten. Und wenn Patienten die Hintergründe der antibiotischen Therapie verstehen, sind Therapietreue und Zufriedenheit deutlich besser. Einige Punkte sollte das Praxisteam immer wieder erklären - etwa dass die Antibiotika solange eingenommen werden müssen, wie es der Arzt verordnet hat, damit keine der Erreger überleben und sich Resistenzen gegenüber dem Antibiotikum bilden können. Der Beitrag in der Rubrik „sprechstunde" beschäftigt sich in dieser Ausgabe daher mit der „rationellen Antibiotikatherapie“.

Dr. Reinhard Merz

\section{„info praxisteam“ im Web}

Unser MFA-Magazin erscheint viermal jährlich. Alle Beiträge sind unter

$$
\text { www.info-praxisteam.de }
$$

auch im Internet abrufbar und können direkt kommentiert werden.

Auch für mobile Endgeräte wie Smartphones oder Tablets ist "info praxisteam" verfügbar. Die App wird sowohl für iOS von Apple als auch für Geräte mit Android-Plattform angeboten. Mehr darüber, wie man die App beziehen kann, erfahren Sie auf der oben angegebenen Website.

Zu den Beiträgen im Heft gibt es von der Redaktion ausgesuchte Verweise auf weiterführende Informationen online. Viele davon sind auch für Sie als Arzt interessant.

Sie erreichen uns per E-Mail unter:

redaktion@info-praxisteam.de 